\title{
Functions of locally bounded variation on Wiener spaces
}

\author{
Masanori Hino \\ Graduate School of Engineering Science, Osaka University \\ hino@sigmath.es.osaka-u.ac.jp
}

We introduce the concept of functions of locally bounded variation on abstract Wiener spaces and study their properties. Some nontrivial examples and applications to stochastic analysis are also discussed.

\section{Introduction}

Functions of bounded variation (BV functions) on abstract Wiener spaces were first studied in $[5 ; 6]$ for their applications in stochastic analysis, but recently BV functions on infinite dimensional spaces have attracted attention for a variety of reasons. In this paper, we newly introduce the space $B V_{\text {loc }}$ of functions of locally bounded variation (local $\mathrm{BV}$ functions) on the abstract Wiener space $(E, H, \mu)$. Needless to say, there could be several ways of localizing the concept of bounded variation. In this article, we adopt the ideas in the theory of (quasi-)regular Dirichlet forms, which are suitable for application to stochastic analysis. Indeed, we show that a Dirichlet form of type

$$
\mathcal{E}^{\rho}(f, g)=\frac{1}{2} \int_{E}\langle\nabla f, \nabla g\rangle_{H} \rho d \mu
$$

associated with a nonnegative function $\rho$ in $\dot{B} V_{\text {loc }}$ with some extra assumptions provides a diffusion process that has the Skorokhod representation (Theorem 10). This result is regarded as a natural generalization of $[6$, Theorem 4.2], where $\rho$ is assumed to be a BV function instead. We also consider the classical Wiener space on $\mathbb{R}^{d}$ as $E$ and provide a sufficient condition for an open set $O$ of $\mathbb{R}^{d}$ so that the indicator function of the set of all paths staying in the closure $\bar{O}$ is a local BV function. Accordingly, we can construct the (modified) reflecting Ornstein-Uhlenbeck process with 
the Skorokhod representation on the set of paths staying in $\bar{O}$ under a rather weak condition on $O$. This is a study related to another paper [9], in which a sufficient condition was given for the above-mentioned indicator function to be a BV function on either pinned path spaces or one-sided pinned spaces.

The remainder of this paper is organized as follows. In Section 2, we introduce the concept of local BV functions on a Wiener space and study their properties. In Section 3, we provide a sufficient condition for a class of indicator functions to be local BV functions.

\section{The space $\dot{B} V_{\text {loc }}$ on Wiener spaces}

Let $(E, H, \mu)$ be an abstract Wiener space. That is, $E$ is a separable Banach space, $H$ is a separable Hilbert space densely and continuously embedded in $E$, and $\mu$ is a Gaussian measure on $E$ that satisfies

$$
\int_{E} \exp (\sqrt{-1} l(z)) \mu(d z)=\exp \left(-|l|_{H}^{2} / 2\right), \quad l \in E^{*} .
$$

Here, we regard the topological dual of $E, E^{*}$, as a dense subspace of $H$ by the natural identification $H^{*} \simeq H$. The inner product and the norm of $H$ are denoted by $\langle\cdot, \cdot\rangle_{H}$ and $|\cdot|_{H}$, respectively. We set

$$
\mathcal{F} C_{b}^{1}=\left\{\begin{array}{l|l}
u: E \rightarrow \mathbb{R} & \begin{array}{l}
u(z)=f\left(l_{1}(z), \ldots, l_{m}(z)\right), l_{1}, \ldots, l_{m} \in E^{*}, \\
f \in C_{b}^{1}\left(\mathbb{R}^{m}\right) \text { for some } m \in \mathbb{N}
\end{array}
\end{array}\right\}
$$

and define $\mathcal{F} C_{b}^{1}\left(E^{*}\right)$ as the set of all linear combinations of $H$-valued functions $u(\cdot) l$ on $E$ with $u \in \mathcal{F} C_{b}^{1}$ and $l \in E^{*} \subset H$. For $u \in \mathcal{F} C_{b}^{1}, \nabla u$ denotes the $H$-derivative of $u$, which is an $H$-valued function on $E$ that is characterized by the identity

$$
\langle\nabla u(z), l\rangle_{H}=\lim _{\varepsilon \rightarrow 0}(u(z+\varepsilon l)-u(z)) / \varepsilon, \quad l \in E^{*} \subset H \subset E .
$$

In the same way, $\nabla u$ is defined for $u \in \mathcal{F} C_{b}^{1}\left(E^{*}\right)$ as an $H \otimes H$-valued function on $E$.

Let $(X, \mathcal{X}, \nu)$ be a measure space and let $Y$ be a separable Hilbert space with norm $|\cdot|_{Y}$. For $p \in[1,+\infty]$, we denote by $L^{p}(X \rightarrow Y ; \nu)$ the space of all $Y$-valued $L^{p}$-functions on $(X, \mathcal{X}, \nu)$ with the norm defined as

$$
\|f\|_{L^{p}(\nu)}= \begin{cases}\left(\int_{X}|f(x)|_{Y}^{p} \nu(d x)\right)^{1 / p}, & p \in[1,+\infty), \\ \nu-\operatorname{essup}_{x \in E}|f(x)|_{Y}, & p=+\infty\end{cases}
$$


As usual, two functions that are equal a.e. are identified. If $X=E$ or $\nu=\mu$ or $Y=\mathbb{R}$, we often omit these symbols from the notation. In particular, $L^{p}$ and $\|\cdot\|_{p}$ represent $L^{p}(E ; \mu)$ and $\|\cdot\|_{L^{p}(\mu)}$, respectively. For $p \in[1,+\infty)$, the closure of $\mathcal{F} C_{b}^{1}\left(\right.$ resp. $\left.\mathcal{F} C_{b}^{1}\left(E^{*}\right)\right)$ with respect to the norm $\left(\|\cdot\|_{p}^{p}+\|\nabla \cdot\|_{p}^{p}\right)^{1 / p}$ will be denoted by $\mathbb{D}^{1, p}\left(\right.$ resp. $\left.\mathbb{D}^{1, p}(H)\right)$. The operator $\nabla$ extends to a continuous map from $\mathbb{D}^{1, p}$ to $L^{p}(E \rightarrow H)$. Let $\nabla^{*}$ denote the adjoint operator of $\nabla$. This is regarded as a bounded operator from $\mathbb{D}^{1, p}(H)$ to $L^{p}$ for $p \in(1, \infty)$. We note that both $\nabla$ and $\nabla^{*}$ have the local property in the sense that, if $f \in \mathbb{D}^{1,2}$ (resp. $G \in \mathbb{D}^{1,2}(H)$ ) satisfies $f=0$ (resp. $G=0$ ) $\mu$-a.e. on some measurable set $A$, then $\nabla f=0$ (resp. $\left.\nabla^{*} G=0\right) \mu$-a.e. on $A$. See, e.g., [11, Propositions 1.3.16 and 1.3.15] for the proof.

Let $L(\log L)^{1 / 2}$ denote the set of all real-valued $\mu$-measurable functions $f$ on $E$ such that $f(0 \vee \log |f|)^{1 / 2} \in L^{1}$. For $\rho \in L(\log L)^{1 / 2}$, define

$$
\begin{aligned}
& V(\rho)=\sup \left\{\left.\int_{E}\left(\nabla^{*} G\right) \rho d \mu\left|G \in \mathcal{F} C_{b}^{1}\left(E^{*}\right),\right| G(z)\right|_{H} \leq 1 \text { for every } z \in E\right\} \\
& (\leq+\infty) .
\end{aligned}
$$

The function space $B V$ on $E$ is defined as

$$
B V=\left\{\rho \in L(\log L)^{1 / 2} \mid V(\rho)<\infty\right\} .
$$

A function in $B V$ is called a BV function or a function of bounded variation. We remark that the following inequality holds for $p \in(1,+\infty]$ and $\rho \in$ $B V \cap L^{p}$.

$$
\int_{E}\left(\nabla^{*} G\right) \rho d \mu \leq V(\rho)\|G\|_{\infty}, \quad G \in \mathbb{D}^{1, q}(H),
$$

where $q$ is the conjugate exponent of $p$.

For a function space $\mathcal{C}$ on $E$ and a $\mu$-measurable subset $A$ of $E, \mathcal{C}_{A}$ denotes the space of all functions in $\mathcal{C}$ vanishing $\mu$-a.e. on $E \backslash A$, and $\mathcal{C}_{b}$ denotes the set of all bounded functions in $\mathcal{C}$. Moreover, $\mathcal{C}_{A, b}$ denotes $\mathcal{C}_{A} \cap \mathcal{C}_{b}$

For $\xi \in L^{1}, F^{\xi}$ denotes the support of the measure $|\xi| \cdot \mu$. For $\xi \in L^{1}$ with $\xi \geq 0 \mu$-a.e., we define a bilinear form $\left(\mathcal{E}, \mathcal{F} C_{b}^{1}\right)$ on $L^{2}\left(F^{\xi}, \xi \cdot \mu\right)$ by

$$
\mathcal{E}^{\xi}(f, g)=\frac{1}{2} \int_{E}\langle\nabla f, \nabla g\rangle_{H} \xi d \mu, \quad f, g \in \mathcal{F} C_{b}^{1} .
$$

The set of all $\xi$ such that $\left(\mathcal{E}, \mathcal{F} C_{b}^{1}\right)$ is closable on $L^{2}\left(F^{\xi} ; \xi \cdot \mu\right)$ is denoted by $Q R$. For $\xi \in Q R$, the closure of $\left(\mathcal{E}, \mathcal{F} C_{b}^{1}\right)$, denoted by $\left(\mathcal{E}^{\xi}, \mathcal{F}^{\xi}\right)$, is a quasiregular Dirichlet form on $L^{2}\left(F^{\xi} ; \xi \cdot \mu\right)$ (see, e.g., [12] and [5, Theorem 2.1] 
for the proof). The space $\mathcal{F}^{\xi}$ is regarded as a Hilbert space by using the inner product $(f, g) \mapsto \mathcal{E}^{\xi}(f, g)+\int_{F^{\xi}} f g \xi d \mu$. The associated capacity Cap ${ }^{\xi}$ is then defined as

$$
\operatorname{Cap}^{\xi}(A)=\inf \left\{\begin{array}{l|l}
\mathcal{E}^{\xi}(f, f)+\|f\|_{L^{2}(\xi \cdot \mu)}^{2} & \begin{array}{l}
f \in \mathcal{F}^{\xi} \text { and } f \geq 1 \xi \cdot \mu \text {-a.e. on } \\
\text { some open set including } A
\end{array}
\end{array}\right\}
$$

for $A \subset F^{\xi}$. The concept " $\mathcal{E}^{\xi}$-quasi-everywhere" ( $\mathcal{E}^{\xi}$-q.e.) is based on this capacity. An increasing sequence $\left\{F_{k}\right\}_{k=1}^{\infty}$ of closed sets in $F^{\xi}$ is called an $\mathcal{E}^{\xi}$-nest if $\lim _{k \rightarrow \infty} \operatorname{Cap}^{\xi}\left(F^{\xi} \backslash F_{k}\right)=0$. It is known that $\left\{F_{k}\right\}_{k=1}^{\infty}$ is an $\mathcal{E}^{\xi}$-nest if and only if $\bigcup_{k=1}^{\infty} \mathcal{F}_{F_{k}}^{\xi}$ is dense in $\mathcal{F}^{\xi}$. In this situation, the set $S^{\xi}$ of all smooth measures is described as the totality of all positive Borel measures $\nu$ on $F^{\xi}$ such that $\nu$ charges no set of zero capacity Cap ${ }^{\xi}$ and there exists an $\mathcal{E}^{\xi}$-nest $\left\{F_{k}\right\}_{k=1}^{\infty}$ such that $\nu\left(F_{k}\right)<\infty$ for all $k$. A function $f$ on $E$ is called $\mathcal{E}^{\xi}$-quasi-continuous if there exists an $\mathcal{E}^{\xi}$-nest $\left\{F_{k}\right\}_{k=1}^{\infty}$ such that $\left.f\right|_{F_{k}}$ is continuous on $F_{k}$ for every $k$. Any function $f \in \mathcal{F}^{\xi}$ has an $\mathcal{E}^{\xi}$-quasi-continuous modification $\tilde{f}$. When $\xi \equiv 1$, we write $\mathcal{E}, \mathcal{F}$, and Cap instead of $\mathcal{E}^{\xi}, \mathcal{F}^{\xi}$, and $\mathrm{Cap}^{\xi}$, respectively. We note that $\mathcal{F}=\mathbb{D}^{1,2}$. For a subset $A$ of $E, e_{A}$ denotes the equilibrium potential of $A$ with respect to $(\mathcal{E}, \mathcal{F})$ : that is, $e_{A}$ attains the infimum of $\{\mathcal{E}(f, f)+$ $\|f\|_{2}^{2} \mid f \in \mathbb{D}^{1,2}$ and $\tilde{f} \geq 1 \mathcal{E}$-q.e. on $\left.A\right\}$, which is equal to $\operatorname{Cap}(A)$ (cf. [7, Theorem 2.1.5]). Henceforth, we will always assume that $e_{A}$ is $\mathcal{E}$-quasicontinuous by itself. Any $G \in \mathbb{D}^{1,2}(H)$ also has an $\mathcal{E}$-quasi-continuous modification $\tilde{G}$ (cf. [2, Chapter VII, Theorem 1.3.1]).

One of the fundamental properties of BV functions is given by the following theorem.

Theorem 1 (cf. [6, Theorem 3.9]). For $\rho \in B V$, there exists a positive finite measure $\nu$ on $E$ and an $H$-valued Borel function $\sigma$ on $E$ such that $|\sigma|_{H}=1 \nu$-a.e., and for every $G \in \mathcal{F} C_{b}^{1}\left(E^{*}\right)$,

$$
\int_{E}\left(\nabla^{*} G\right) \rho d \mu=\int_{E}\langle G, \sigma\rangle_{H} d \nu
$$

The measure $\nu$ belongs to $S^{|\rho|+1}$. If $\rho \in Q R$ in addition, then $\left.\nu\right|_{E \backslash F^{\rho}}=0$ and $\left.\nu\right|_{F^{\rho}} \in S^{\rho}$. Also, $\nu$ and $\sigma$ are uniquely determined in the following sense: if $\nu^{\prime}$ and $\sigma^{\prime}$ are another pair satisfying (2) for all $G \in \mathcal{F} C_{b}^{1}\left(E^{*}\right)$, then $\nu=\nu^{\prime}$ and $\sigma=\sigma^{\prime} \nu$-a.e.

We now introduce some localized function spaces.

Definition 2. Let $\rho$ be a real-valued $\mu$-measurable function on $E$. 
(i) For $p \in[1,+\infty]$, we say $\rho \in \dot{L}_{\text {loc }}^{p}$ if there exists an $\mathcal{E}$-nest $\left\{F_{k}\right\}_{k=1}^{\infty}$ such that $\rho \cdot \mathbf{1}_{F_{k}} \in L^{p}$ for every $k \in \mathbb{N}$.

(ii) We say $\rho \in \dot{B} V_{\text {loc }}$ if there exists an $\mathcal{E}$-nest $\left\{F_{k}\right\}_{k=1}^{\infty}$ and $\left\{\rho_{k}\right\}_{k=1}^{\infty} \subset B V$ such that $\rho=\rho_{k} \mu$-a.e. on $F_{k}$ for every $k \in \mathbb{N}$.

The following are some properties of $\dot{B} V_{\text {loc }}$.

Theorem 3. For $\rho \in \dot{L}_{\mathrm{loc}}^{2}$, the implications $(\mathrm{a}) \Leftrightarrow(\mathrm{b}) \Leftarrow(\mathrm{c})$ hold.

(a) $\rho \in \dot{B} V_{\text {loc }}$.

(b) There exists an $\mathcal{E}$-nest $\left\{F_{k}\right\}_{k=1}^{\infty}$ and positive numbers $\left\{c_{k}\right\}_{k=1}^{\infty}$ such that for every $k \in \mathbb{N}, \rho \cdot \mathbf{1}_{F_{k}} \in L^{2}$ and

$$
\int_{E} \rho \nabla^{*} G d \mu \leq c_{k}\|G\|_{\infty}, \quad G \in \mathbb{D}^{1,2}(H)_{F_{k}, b}
$$

(c) There exists an $\mathcal{E}$-nest $\left\{F_{k}\right\}_{k=1}^{\infty}$ and $\left\{f_{m}\right\}_{m=1}^{\infty} \subset \mathbb{D}^{1,1}$ such that for every $k \in \mathbb{N},\left(f_{m}-\rho\right) \cdot \mathbf{1}_{F_{k}}$ converges to 0 in $L^{1}$ as $m \rightarrow \infty$ and $\left\|\left(\nabla f_{m}\right) \cdot \mathbf{1}_{F_{k}}\right\|_{1}$ is bounded in $\mathrm{m}$.

Unlike the case of finite-dimensional spaces, we must take care with the localized test functions on $E$. Indeed, except for the zero function there are no functions $f$ in $\mathcal{F} C_{b}^{1}$ such that $f$ vanishes outside a bounded set. This is why we introduce $\mathbb{D}^{1,2}(H)_{F_{k}, b}$ in the theorem above.

For the proof of Theorem 3, we introduce some lemmas.

Lemma 4. Let $\left\{F_{k}\right\}_{k=1}^{\infty}$ be an $\mathcal{E}$-nest. Then, there exists another $\mathcal{E}$-nest $\left\{F_{k}^{\prime}\right\}_{k=1}^{\infty}$ and functions $\left\{\varphi_{k}\right\}_{k=1}^{\infty} \subset \bigcup_{n=1}^{\infty} \mathbb{D}_{F_{n}}^{1,2}$ such that for any $k, F_{k}^{\prime} \subset F_{l}$ for some $l \geq k, \varphi_{k}$ is $\mathcal{E}$-quasi-continuous, $0 \leq \varphi_{k} \leq 1$ on $E, \varphi_{k}=1$ on $F_{k}^{\prime}$, and $\lim _{k \rightarrow \infty} \mathcal{E}\left(\varphi_{k}, \varphi_{k}\right)=0$.

Proof. We denote $E \backslash F_{k}$ by $F_{k}^{c}$, etc. Take an increasing sequence $\{n(k)\}_{k=1}^{\infty}$ of natural numbers such that $\operatorname{Cap}\left(F_{n(k)}^{c}\right)<2^{-k}$ for every $k$. There exists an $\mathcal{E}$-nest $\left\{\hat{F}_{l}\right\}_{l=1}^{\infty}$ such that $e_{F_{n(k)}^{c}}$ is continuous on each $\hat{F}_{l}$ for every $k$ (cf. [7, Theorem 2.1.2]). For $k \in \mathbb{N}$, let $\varphi_{k}=1 \wedge 2\left(1-e_{F_{n(k)}^{c}}\right)$ and $\check{F}_{k}=\left\{\varphi_{k}=1\right\}$. Since $\check{F}_{k}^{c}=\left\{e_{F_{n(k)}^{c}}>1 / 2\right\}$,

$$
\operatorname{Cap}\left(\check{F}_{k}^{c}\right) \leq \mathcal{E}\left(2 e_{F_{n(k)}^{c}}, 2 e_{F_{n(k)}^{c}}\right)+\left\|2 e_{F_{n(k)}^{c}}\right\|_{2}^{2}=4 \operatorname{Cap}\left(F_{n(k)}^{c}\right)<2^{-k+2} .
$$

Define $F_{k}^{\prime}=\bigcap_{l=k}^{\infty}\left(\check{F}_{l} \cap \hat{F}_{l} \cap F_{n(l)}\right)$. Then, $\left\{F_{k}^{\prime}\right\}_{k=1}^{\infty}$ is a nondecreasing 
sequence of closed sets and

$$
\begin{aligned}
\operatorname{Cap}\left(\left(F_{k}^{\prime}\right)^{c}\right) & =\operatorname{Cap}\left(\left(\bigcup_{l=k}^{\infty} \check{F}_{l}^{c}\right) \cup \hat{F}_{k}^{c} \cup F_{n(k)}^{c}\right) \\
& \leq \sum_{l=k}^{\infty} \operatorname{Cap}\left(\check{F}_{l}^{c}\right)+\operatorname{Cap}\left(\hat{F}_{k}^{c}\right)+\operatorname{Cap}\left(F_{n(k)}^{c}\right) \\
& \leq 2^{-k+3}+\operatorname{Cap}\left(\hat{F}_{k}^{c}\right)+\operatorname{Cap}\left(F_{n(k)}^{c}\right),
\end{aligned}
$$

which converges to 0 as $k \rightarrow \infty$. Furthermore, $\mathcal{E}\left(\varphi_{k}, \varphi_{k}\right) \leq$ $4 \mathcal{E}\left(e_{F_{n(k)}^{c}}, e_{F_{n(k)}^{c}}\right)<2^{-k+2} \rightarrow 0$ as $k \rightarrow \infty$.

Lemma 5. Let $p \in(1,+\infty]$ and $q$ denote the conjugate exponent of $p$.

(i) Let $f \in B V \cap L^{p}$ and $g \in \mathbb{D}_{b}^{1, q}$. Then, $f g \in B V$ and $V(f g) \leq$ $V(f)\|g\|_{\infty}+\|f \nabla g\|_{1}$.

(ii) Let $f \in B V$ and $g \in \mathbb{D}_{F, b}^{1, q}$ for some $\mu$-measurable set $F \subset E$. If $f \cdot \mathbf{1}_{F} \in L^{p}$, then $f g \in B V \cap L^{p}$ and $V(f g) \leq V(f)\|g\|_{\infty}+\|f \nabla g\|_{1}$.

Proof. (i) Let $\left\{T_{t}\right\}_{t>0}$ be the Ornstein-Uhlenbeck semigroup on $E$. For $t>0, T_{t} f \in \mathbb{D}^{1, p}$, thus $\left(T_{t} f\right) g \in \mathbb{D}^{1,1}$ and

$$
\begin{aligned}
\left\|\nabla\left(\left(T_{t} f\right) g\right)\right\|_{1} & \leq\left\|\nabla\left(T_{t} f\right)\right\|_{1}\|g\|_{\infty}+\left\|\left(T_{t} f\right) \nabla g\right\|_{1} \\
& \leq e^{-t} V(f)\|g\|_{\infty}+\left\|\left(T_{t} f\right) \nabla g\right\|_{1},
\end{aligned}
$$

from, e.g., [6, Proposition 3.6]. Since $\lim _{t \rightarrow 0}\left(T_{t} f\right) g=f g$ in $L^{1}$ and $\lim _{t \rightarrow 0}\left(T_{t} f\right) \nabla g=f \nabla g$ in $L^{1}(H)$, Theorem 3.7 of [6] completes the proof.

(ii) For $n \in \mathbb{N}$, let $f_{n}=(-n) \vee(f \wedge n)$. From [6, Corollary 3.8], $f_{n} \in B V$ and $V\left(f_{n}\right) \leq V(f)$. By the assumption and (i), $f_{n} g \in B V \cap L^{p}$, $\left\|f_{n} g\right\|_{p} \leq\|f g\|_{p}$, and

$$
V\left(f_{n} g\right) \leq V\left(f_{n}\right)\|g\|_{\infty}+\left\|f_{n} \nabla g\right\|_{1} \leq V(f)\|g\|_{\infty}+\|f \nabla g\|_{1},
$$

which is bounded for each $n$. Since $\lim _{n \rightarrow \infty} f_{n} g=f g$ in $L^{1}$, the claim follows.

Lemma 6. For $\rho \in \dot{B} V_{\text {loc }} \cap \dot{L}^{2}$, there exists an $\mathcal{E}$-nest $\left\{F_{k}\right\}_{k=1}^{\infty}$ and functions $\left\{\rho_{k}\right\}_{k=1}^{\infty}$ in $B V \cap L^{2}$ such that $\rho=\rho_{k}$ on $F_{k}$ for every $k \in \mathbb{N}$.

Proof. There exist an $\mathcal{E}$-nest $\left\{F_{k}\right\}_{k=1}^{\infty}$ and $\left\{\rho_{k}\right\}_{k=1}^{\infty} \subset B V$ such that for every $k, \rho \cdot \mathbf{1}_{F_{k}} \in L^{2}$ and $\rho=\rho_{k}$ on $F_{k}$. Take the $\mathcal{E}$-nest $\left\{F_{k}^{\prime}\right\}_{k=1}^{\infty}$ and the functions $\left\{\varphi_{k}\right\}_{k=1}^{\infty}$ in Lemma 4. From Lemma 5 , the assertion is true by taking $\left\{F_{k}^{\prime}\right\}_{k=1}^{\infty}$ and $\left\{\rho \varphi_{k}\right\}_{k=1}^{\infty}$ as $\left\{F_{k}\right\}_{k=1}^{\infty}$ and $\left\{\rho_{k}\right\}_{k=1}^{\infty}$, respectively. 
Proof of Theorem 3. (a) $\Rightarrow(\mathrm{b})$ : Take the $\mathcal{E}$-nest $\left\{F_{k}\right\}_{k=1}^{\infty}$ and $\left\{\rho_{k}\right\}_{k=1}^{\infty} \subset$ $B V \cap L^{2}$ as in Lemma 6 . Let $k \in \mathbb{N}$ and take $G \in \mathbb{D}^{1,2}(H)_{F_{k}, b}$. Since $\nabla^{*} G=0 \mu$-a.e. on $E \backslash F_{k}$,

$$
\int_{E} \rho \nabla^{*} G d \mu=\int_{E} \rho_{k} \nabla^{*} G d \mu \leq V\left(\rho_{k}\right)\|G\|_{\infty},
$$

where the last inequality follows from (1). Thus, (b) holds.

$(\mathrm{b}) \Rightarrow(\mathrm{a})$ : Take the $\mathcal{E}$-nest $\left\{F_{k}^{\prime}\right\}_{k=1}^{\infty}$ and the functions $\left\{\varphi_{k}\right\}_{k=1}^{\infty}$ as in Lemma 4. For $k \in \mathbb{N}$, take $l \in \mathbb{N}$ such that $\varphi_{k} \in \mathbb{D}_{F_{l}}^{1,2}$ and let $\rho_{k}=\rho \varphi_{k}$. Then, $\rho_{k} \in L^{2}$ and $\rho_{k}=\rho$ on $F_{k}^{\prime}$. Moreover, for any $G \in \mathcal{F} C_{b}^{1}\left(E^{*}\right)$,

$$
\begin{aligned}
\int_{E} \rho_{k} \nabla^{*} G d \mu & =\int_{E} \rho \varphi_{k} \nabla^{*} G d \mu \\
& =\int_{E} \rho\left\{\nabla^{*}\left(\varphi_{k} G\right)+\left\langle\nabla \varphi_{k}, G\right\rangle_{H}\right\} d \mu \\
& \leq c_{l}\left\|\varphi_{k} G\right\|_{\infty}+\left\|\rho \cdot \mathbf{1}_{F_{l}}\right\|_{2}\left\|\nabla \varphi_{k}\right\|_{2}\|G\|_{\infty} \\
& \leq\left(c_{l}+\left\|\rho \cdot \mathbf{1}_{F_{l}}\right\|\left\|_{2}\right\| \nabla \varphi_{k} \|_{2}\right)\|G\|_{\infty} .
\end{aligned}
$$

Therefore, $\rho_{k} \in B V$.

$(\mathrm{c}) \Rightarrow(\mathrm{b})$ : We may assume that $\rho \cdot \mathbf{1}_{F_{k}} \in L^{2}$ for every $k$. For $M>0$, define $\Phi_{M}(t)=(-M) \vee(t \wedge M)$ for $t \in \mathbb{R}$. Then, for $k \in \mathbb{N}$ and $G \in$ $\mathbb{D}^{1,2}(H)_{F_{k}, b}$,

$$
\begin{aligned}
\int_{E} \Phi_{M}\left(f_{m}\right) \nabla^{*} G d \mu & =\int_{E}\left\langle\nabla\left(\Phi_{M}\left(f_{m}\right)\right), G\right\rangle_{H} d \mu \\
& \leq\left\|\left(\nabla f_{m}\right) \cdot \mathbf{1}_{F_{k}}\right\|_{1}\|G\|_{\infty} \leq c_{k}\|G\|_{\infty},
\end{aligned}
$$

where $c_{k}:=\sup _{m \in \mathbb{N}}\left\|\left(\nabla f_{m}\right) \cdot \mathbf{1}_{F_{k}}\right\|_{1}<\infty$. Since $\nabla^{*} G \in L^{2}$,

$$
\lim _{m \rightarrow \infty} \int_{E} \Phi_{M}\left(f_{m}\right) \nabla^{*} G d \mu=\int_{E} \Phi_{M}(\rho) \nabla^{*} G d \mu .
$$

Since $\lim _{M \rightarrow \infty}\left(\Phi_{M}(\rho)-\rho\right) \cdot \mathbf{1}_{F_{k}}=0$ in $L^{2}$,

$$
\lim _{M \rightarrow \infty} \int_{E} \Phi_{M}(\rho) \nabla^{*} G d \mu=\int_{E} \rho \nabla^{*} G d \mu .
$$

Therefore, we have $\int_{E} \rho \nabla^{*} G d \mu \leq c_{k}\|G\|_{\infty}$.

The following are some basic lemmas that are used later.

Lemma 7. The following claims hold.

(i) For $\rho \in L^{\infty}, \mathcal{F}^{|\rho|+1}=\mathbb{D}^{1,2}$ and their norms are equivalent. 
(ii) Let $\rho \in Q R \cap L^{\infty}$ and $\left\{F_{k}\right\}_{k=1}^{\infty}$ be an $\mathcal{E}$-nest. Then, $\left.\mathbb{D}^{1,2}\right|_{F^{\rho}}$ is continuously embedded in $\mathcal{F}^{\rho}$ and there exists $c>0$ such that $\operatorname{Cap}^{\rho}(A) \leq$ $c \operatorname{Cap}(A)$ for any $A \subset F^{\rho}$. In particular, $\left\{F_{k} \cap F^{\rho}\right\}_{k=1}^{\infty}$ is an $\mathcal{E}^{\rho}$-nest. Moreover, $\left.\bigcup_{k=1}^{\infty} \mathbb{D}_{F_{k}, b}^{1,2}\right|_{F^{\rho}}$ is dense in $\mathcal{F}^{\rho}$.

Proof. We prove only the last claim of (ii). It suffices to prove that for any function $f \in \mathcal{F} C_{b}^{1},\left.f\right|_{F^{\rho}}$ can be approximated by elements of $\left.\bigcup_{k=1}^{\infty} \mathbb{D}_{F_{k}, b}^{1,2}\right|_{F^{\rho}}$ in $\mathcal{F}^{\rho}$. Since $\bigcup_{k=1}^{\infty} \mathbb{D}_{F_{k}, b}^{1,2}$ is dense in $\mathbb{D}^{1,2}$, there exists $\left\{f_{n}\right\}_{n=1}^{\infty}$ in $\bigcup_{k=1}^{\infty} \mathbb{D}_{F_{k}, b}^{1,2}$ such that $f_{n}$ converges to $f$ in $\mathbb{D}^{1,2}$. Then, $\left.f_{n}\right|_{F^{\rho}}$ converges to $\left.f\right|_{F^{\rho}}$ in $\mathcal{F}^{\rho}$.

Lemma 8. Let $\left\{F_{k}\right\}_{k=1}^{\infty}$ be an $\mathcal{E}$-nest. Let $\nu$ and $\nu^{\prime}$ be $\mathcal{E}$-smooth measures on $E$ such that $\nu\left(F_{k}\right)<\infty$ and $\nu^{\prime}\left(F_{k}\right)<\infty$ for every $k \in \mathbb{N}$. Let $\sigma$ and $\sigma^{\prime}$ be $H$-valued Borel functions on $E$ such that $|\sigma|_{H}=1 \nu$-a.e. and $\left|\sigma^{\prime}\right|_{H}=1$ $\nu^{\prime}$-a.e. If $\int_{E}\langle\tilde{G}, \sigma\rangle_{H} d \nu=\int_{E}\left\langle\tilde{G}, \sigma^{\prime}\right\rangle_{H} d \nu^{\prime}$ for every $G \in \bigcup_{k=1}^{\infty} \mathbb{D}^{1,2}(H)_{F_{k}, b}$, then $\nu=\nu^{\prime}$ and $\sigma=\sigma^{\prime} \nu$-a.e.

Proof. Let $\xi=\nu+\nu^{\prime}$ and $\gamma=\sigma \frac{d \nu}{d \xi}-\sigma^{\prime} \frac{d \nu^{\prime}}{d \xi}$. Then, $\int_{E}\langle\tilde{G}, \gamma\rangle_{H} d \xi=0$ for every $G \in \bigcup_{k=1}^{\infty} \mathbb{D}_{F_{k}, b}^{1,2}$. Taking a uniformly bounded sequence $\left\{G_{n}\right\}_{n=1}^{\infty}$ from $\bigcup_{k=1}^{\infty} \mathbb{D}_{F_{k}, b}^{1,2}$ such that $\left\langle\tilde{G}_{n}, \gamma\right\rangle_{H} \rightarrow|\gamma|_{H} \xi$-a.e. as $n \rightarrow \infty$, we obtain $\gamma=0$-a.e. Therefore, $|\sigma|_{H} \frac{d \nu}{d \xi}=\left|\sigma^{\prime}\right|_{H} \frac{d \nu^{\prime}}{d \xi} \xi$-a.e. Since $|\sigma|_{H}=1 \nu$-a.e., $|\sigma|_{H} \frac{d \nu}{d \xi}=\frac{d \nu}{d \xi} \xi$-a.e. Similarly, $\left|\sigma^{\prime}\right|_{H} \frac{d \nu^{\prime}}{d \xi}=\frac{d \nu^{\prime}}{d \xi} \xi$-a.e. Then, $\frac{d \nu}{d \xi}=\frac{d \nu^{\prime}}{d \xi} \xi$-a.e., which implies $\nu=\nu^{\prime}$. The identity $\sigma=\sigma^{\prime} \nu$-a.e. follows from $\gamma=0 \xi$-a.e. and $\nu=\nu^{\prime}$.

Theorem 9. For $\rho \in \dot{B} V_{\text {loc }} \cap \dot{L}^{2}$, there exist an $H$-valued Borel function $\sigma$ on $E$, an $\mathcal{E}$-smooth measure $\nu$ on $E$, and an $\mathcal{E}$-nest $\left\{F_{k}\right\}_{k=1}^{\infty}$ such that $\rho \cdot \mathbf{1}_{F_{k}} \in L^{2}$ and $\nu\left(F_{k}\right)<\infty$ for every $k \in \mathbb{N},|\sigma|_{H}=1 \nu$-a.e., and

$$
\int_{E} \rho \nabla^{*} G d \mu=\int_{E}\langle\tilde{G}, \sigma\rangle_{H} d \nu \quad \text { for every } G \in \bigcup_{k=1}^{\infty} \mathbb{D}^{1,2}(H)_{F_{k}, b}
$$

where $\tilde{G}$ denotes an $\mathcal{E}$-quasi-continuous modification of $G$. The pair $\nu$ and $\sigma$ is uniquely determined in the following sense: if another pair $\nu^{\prime}$ and $\sigma^{\prime}$ with some $\mathcal{E}$-nest satisfies the conditions above, then $\nu=\nu^{\prime}$ and $\sigma=\sigma^{\prime}$ $\nu$-a.e.

$$
\text { If } \rho \in Q R \cap L^{\infty} \text { in addition, then }\left.\nu\right|_{E \backslash F^{\rho}}=0 \text { and }\left.\nu\right|_{F^{\rho}} \in S^{\rho} \text {. }
$$

Proof. Take an $\mathcal{E}$-nest $\left\{F_{k}\right\}_{k=1}^{\infty}$ and $\left\{\rho_{k}\right\}_{k=1}^{\infty} \subset B V \cap L^{2}$ as in Lemma 6 . From Theorem 1, for each $k$, there exist an $H$-valued Borel function $\sigma_{k}$ on 
$E$ and an $\mathcal{E}$-smooth finite measure $\nu_{k}$ on $E$ such that $\left|\sigma_{k}\right|_{H}=1 \nu_{k}$-a.e. and

$$
\int_{E} \rho_{k} \nabla^{*} G d \mu=\int_{E}\left\langle G, \sigma_{k}\right\rangle_{H} d \nu_{k}
$$

for every $G \in \mathcal{F} C_{b}^{1}\left(E^{*}\right)$. By approximation, (4) holds for $G \in \mathbb{D}^{1,2}(H)_{b}$ with $\left\langle G, \sigma_{k}\right\rangle_{H}$ replaced by $\left\langle\tilde{G}, \sigma_{k}\right\rangle_{H}$.

Take an $\mathcal{E}$-nest $\left\{F_{k}^{\prime}\right\}_{k=1}^{\infty}$ and functions $\left\{\varphi_{k}\right\}_{k=1}^{\infty}$ as in Lemma 4. For $k \in \mathbb{N}$, let $n(k)$ denote a number such that $\varphi_{k} \in \mathbb{D}_{F_{n(k)}}^{1,2}$. We may assume that $\{n(k)\}_{k=1}^{\infty}$ is an increasing sequence. Then, for $l \geq k$,

$$
\varphi_{k} \cdot \nu_{n(k)}=\varphi_{k} \cdot \nu_{n(l)} \text { and } \sigma_{n(k)}=\sigma_{n(l)}\left(\varphi_{k} \cdot \nu_{n(k)}\right) \text {-a.e. }
$$

Indeed, for any $G \in \mathbb{D}^{1,2}(H)_{b}$, we have $\varphi_{k} G \in \mathbb{D}^{1,2}(H)_{F_{n(k)}, b}$ and

$$
\begin{aligned}
\int_{E} \varphi_{k}\left\langle\tilde{G}, \sigma_{n(k)}\right\rangle_{H} d \nu_{n(k)} & =\int_{E} \rho_{n(k)} \nabla^{*}\left(\varphi_{k} G\right) d \mu \\
& =\int_{E} \rho_{n(l)} \nabla^{*}\left(\varphi_{k} G\right) d \mu \\
& =\int_{E} \varphi_{k}\left\langle\tilde{G}, \sigma_{n(l)}\right\rangle_{H} d \nu_{n(l)}
\end{aligned}
$$

Thus, (5) follows from Lemma 8. Therefore, we can define $\sigma$ and $\nu$ so that $\sigma=\sigma_{n(k)}$ on $F_{k}^{\prime}$ and $\left.\nu\right|_{F_{k}^{\prime}}=\left.\nu_{n(k)}\right|_{F_{k}^{\prime}}$ for $k \in \mathbb{N}$, and $\nu\left(E \backslash \bigcup_{k=1}^{\infty} F_{k}^{\prime}\right)=0$. Then, the conditions described in the theorem are satisfied with $\left\{F_{k}\right\}_{k=1}^{\infty}$ replaced by $\left\{F_{k}^{\prime}\right\}_{k=1}^{\infty}$.

Suppose that another $\nu^{\prime}, \sigma^{\prime}$, and an $\mathcal{E}$-nest $\left\{F_{k}^{\prime \prime}\right\}_{k=1}^{\infty}$ satisfy the required conditions. Applying Lemma 8 to the $\mathcal{E}$-nest $\left\{F_{k} \cap F_{k}^{\prime \prime}\right\}_{k=1}^{\infty}$, we obtain that $\nu=\nu^{\prime}$ and $\sigma=\sigma^{\prime} \nu$-a.e.

Lastly, suppose $\rho \in Q R \cap L^{\infty}$ in addition. Let $l \in E^{*}$ and $g \in \mathbb{D}_{F_{k}, b}^{1,2}$ for some $k \in \mathbb{N}$. Letting $G=g(\cdot) l$ in $(3)$, we obtain

$$
\int_{F^{\rho}}\left(\partial_{l} g+g \cdot l(\cdot)\right) \rho d \mu=\int_{E} \tilde{g}\langle l, \sigma\rangle_{H} d \nu .
$$

Denote the left-hand side of (6) by $I(g)$. Then, $I$ provides a bounded functional on $\mathcal{F}^{\rho}$ as well as on $\mathbb{D}^{1,2}$. From the observation that $I(g)$ does not change if $g$ is replaced by $(-M) \vee(g \wedge M)$ with $M=\mu$-ess sup $\left|g \cdot \mathbf{1}_{F_{k}}\right|$, the inequality $I(g) \leq\left\|\left.g\right|_{F^{\rho}}\right\|_{L^{\infty}(\rho \cdot \mu)}|l|_{H} \nu\left(F_{k}\right)$ holds. Then, from Lemma 7 and [8, Theorem 2.18], there exists a unique $\nu_{l} \in S^{\rho}$ such that $I(g)=\int_{F^{\rho}} \tilde{g} d \nu_{l}$ for all $g \in \bigcup_{k=1}^{\infty} \mathbb{D}_{F_{k}, b}^{1,2}$. From the uniqueness of the integral representation of $I(g)$ as a functional on $\mathbb{D}^{1,2}$, we conclude that $\left.\left(\langle l, \sigma\rangle_{H} \cdot \nu\right)\right|_{E \backslash F^{\rho}}=0$ and $\left.\left(\langle l, \sigma\rangle_{H} \cdot \nu\right)\right|_{F^{\rho}}=\nu_{l} \in S^{\rho}$. Because $l$ is arbitrary, we deduce that $\left.\nu\right|_{E \backslash F^{\rho}}=0$ and $\left.\nu\right|_{F^{\rho}} \in S^{\rho}$. 
We will denote $\nu$ and $\sigma$ in the theorem above by $\|D \rho\|$ and $\sigma_{\rho}$, respectively.

Theorem 10 (Skorokhod representation). Let $\rho \in B V_{\operatorname{loc}} \cap Q R \cap L^{\infty}$. Then the sample path of the diffusion process $\mathbf{M}^{\rho}=\left(X_{t}, \mathcal{M}_{t}, P_{z}\right)$ associated with $\left(\mathcal{E}^{\rho}, \mathcal{F}^{\rho}\right)$ admits the following expression as a sum of three E-valued continuous additive functionals.

$$
\begin{aligned}
& X_{t}(\omega)-X_{0}(\omega) \\
& =W_{t}(\omega)-\frac{1}{2} \int_{0}^{t} X_{s}(\omega) d s+\frac{1}{2} \int_{0}^{t} \sigma_{\rho}\left(X_{s}(\omega)\right) d A_{s}^{\|D \rho\|}(\omega), \quad t \geq 0 .
\end{aligned}
$$

Here, $A^{\|D \rho\|}$ is a real-valued positive continuous additive functional associated with $\|D \rho\|$ via the Revuz correspondence. Moreover, for $\mathcal{E}^{\rho}$-q.e. $z \in F^{\rho}$, $\left\{W_{t}\right\}_{t \geq 0}$ is the $\left\{\mathcal{M}_{t}\right\}$-Brownian motion on $E$ under $P_{z}$.

Proof. The proof is provided along the same lines as those of $[6$, Theorem 4.2] and [5, Theorem 3.2] with the use of [4, Theorem 6.1] instead of $\left[4\right.$, Theorem 6.2] (or [5, Theorem 2.2]). We define $\left\{W_{t}\right\}_{t \geq 0}$ so that (7) holds. Then, $\left\{W_{t}\right\}_{t \geq 0}$ is an $E$-valued continuous additive functional with the same defining and exceptional sets as $A^{\|D \rho\|}$. Take the $\mathcal{E}$-nest $\left\{F_{k}\right\}_{k=1}^{\infty}$ in Theorem 9. Let $l \in E^{*}$ and consider the identity

$$
l\left(X_{t}\right)-l\left(X_{0}\right)=l\left(W_{t}\right)-\frac{1}{2} \int_{0}^{t} l\left(X_{s}\right) d s+\frac{1}{2} \int_{0}^{t}\left\langle l, \sigma_{\rho}\left(X_{s}\right)\right\rangle_{H} d A_{s}^{\|D \rho\|} .
$$

We note that $\left.l(\cdot)\right|_{F^{\rho}} \in \mathcal{F}^{\rho}$. From (3), the identity

$$
\mathcal{E}^{\rho}(l(\cdot), g)=\frac{1}{2} \int_{F^{\rho}} g l(\cdot) \rho d \mu-\frac{1}{2} \int_{F^{\rho}} \tilde{g}\left\langle l, \sigma_{\rho}\right\rangle_{H} d\|D \rho\|
$$

holds for $g \in \bigcup_{k=1}^{\infty} \mathbb{D}_{F_{k}, b}^{1,2}$. Take a nest $\left\{F_{k}^{\prime}\right\}_{k=1}^{\infty}$ and functions $\left\{\varphi_{k}\right\}_{k=1}^{\infty}$ as in Lemma 4. For any $g \in \mathcal{F}_{F_{k}^{\prime}, b}^{\rho}$ with $k \in \mathbb{N}$, there exist $\left\{g_{n}\right\} \subset$ $\bigcup_{m=1}^{\infty} \mathbb{D}_{F_{m}, b}^{1,2}$ such that $\left\{g_{n}\right\}$ are uniformly bounded and $\left.g_{n}\right|_{F^{\rho}}$ converges to $g$ in $\mathcal{F}^{\rho}$ as $n \rightarrow \infty$. Since $\mathcal{E}\left(g_{n} \varphi_{k}, g_{n} \varphi_{k}\right)^{1 / 2} \leq \mathcal{E}\left(g_{n}, g_{n}\right)^{1 / 2}\left\|\varphi_{k}\right\|_{\infty}+$ $\mathcal{E}\left(\varphi_{k}, \varphi_{k}\right)^{1 / 2}\left\|g_{n}\right\|_{\infty}, \quad\left\|g_{n} \varphi_{k}\right\|_{2} \leq\left\|g_{n}\right\|_{\infty}\left\|\varphi_{k}\right\|_{2}$, and $\left.\left(g_{n} \varphi_{k}\right)\right|_{F^{\rho}} \rightarrow g$ in $L^{2}(\rho \cdot \mu)$, the Cesàro means of a certain subsequence of $\left\{\left.\left(g_{n} \varphi_{k}\right)\right|_{F^{\rho}}\right\}_{n=1}^{\infty}$, which are all elements of $\left.\mathbb{D}_{F_{l}, b}^{1,2}\right|_{F^{\rho}}$ for some $l$, converges to $g$ in $\mathcal{F}^{\rho}$. A further suitable subsequence of their $\mathcal{E}^{\rho}$-quasi-continuous modifications converges $\mathcal{E}^{\rho}$-q.e. from [7, Theorem 2.1.4]. Therefore, the above identity holds for $g \in \bigcup_{k=1}^{\infty} \mathcal{F}_{F_{k}^{\prime}, b}^{\rho}$, where $\tilde{g}$ is interpreted as an $\mathcal{E}^{\rho}$-quasicontinuous modification of $g$. From [4, Theorem 6.1], the Fukushima decomposition of $l\left(X_{t}\right)-l\left(X_{0}\right)$ is given by the sum of $M_{t}^{l}:=l\left(W_{t}\right)$ and $N_{t}^{l}:=-\frac{1}{2} \int_{0}^{t} l\left(X_{s}\right) d s+\frac{1}{2} \int_{0}^{t}\left\langle l, \sigma_{\rho}\left(X_{s}\right)\right\rangle_{H} d A_{s}^{\|D \rho\|}$. Moreover, the quadratic 
variation of $\left\{M_{t}^{l}\right\}$ is equal to $\left\{t|l|_{H}^{2}\right\}$. From [6, Lemma 4.1], we conclude that $\left\{W_{t}\right\}_{t \geq 0}$ is the $E$-valued Brownian motion, which completes the proof.

\section{Indicator functions in $\dot{B} V_{\text {loc }}$}

In this section, we provide some nontrivial examples of functions in $\dot{B} V_{\text {loc }}$. Let $d \in \mathbb{N}$ and $T>0$. We consider the classical $d$-dimensional Wiener space as $(E, H, \mu)$ : that is,

$$
\begin{aligned}
& E=\left\{w \in C\left([0, T] \rightarrow \mathbb{R}^{d}\right) \mid w(0)=0\right\}, \\
& H=\left\{h \in E \mid h \text { is absolutely continuous and } \int_{0}^{T}|\dot{h}(s)|_{\mathbb{R}^{d}}^{2} d s<\infty\right\},
\end{aligned}
$$

and $\mu$ is the Wiener measure on $E$. For a subset $A$ of $\mathbb{R}^{d}, \bar{A}$ (resp. $\partial A$ ) denotes the closure (resp. boundary) of $A$, and $A^{c}$ denotes $\mathbb{R}^{d} \backslash A$. Define some subsets of $E$ as follows:

$$
\begin{aligned}
& \Xi_{A}=\{w \in E \mid w(t) \in A \text { for all } t \in[0, T]\}, \\
& \Theta_{A}=\{w \in E \mid w(t) \in A \text { for some } t \in[0, T]\}, \\
& \partial \Xi_{A}=\left\{\begin{array}{l|l}
w \in E & \begin{array}{l}
w(t) \in \bar{A} \text { for all } t \in[0, T] \text { and } \\
w(s) \in \partial A \text { for some } s \in[0, T]
\end{array}
\end{array}\right\}, \\
& \partial^{\prime} \Xi_{A}=\left\{\begin{array}{l|l}
w \in E & \begin{array}{l}
w(t) \in \bar{A} \text { for all } t \in[0, T] \text { and } w(s) \in \partial A \\
\text { for some unique } s \in[0, T]
\end{array}
\end{array}\right\} .
\end{aligned}
$$

We note that $\partial \Xi_{A}$ is the topological boundary of $\Xi_{A}$ in $E$ with the uniform topology. A sufficient condition for $\mathbf{1}_{\Xi_{A}}$ to belong to $B V$ was given in [13; 9]. Here, we provide a sufficient condition for $\mathbf{1}_{\Xi_{A}}$ to belong to $\dot{B} V_{\text {loc }}$.

For $x \in \mathbb{R}^{d}$ and $r \geq 0$, we write $B(x, r)$ and $\bar{B}(x, r)$ for $\left\{z \in \mathbb{R}^{d}\right.$ $\left.|z-x|_{\mathbb{R}^{d}}<r\right\}$ and $\left\{z \in \mathbb{R}^{d}|| z-\left.x\right|_{\mathbb{R}^{d}} \leq r\right\}$, respectively. Let $O$ be a proper open subset of $\mathbb{R}^{d}$ such that $0 \in O$. For $y \in \partial O$, we define $\delta(y) \in[0,+\infty]$ as

$$
\delta(y)=\sup \left\{r \geq 0 \mid \text { there exists } z \in O^{c} \text { such that } \bar{O} \cap \bar{B}(z, r)=\{y\}\right\} .
$$

From $\left[9\right.$, Theorem 5.1], $\mathbf{1}_{\Xi_{\bar{O}}} \in B V$ if the uniform exterior ball condition

$$
\inf _{y \in \partial O} \delta(y)>0
$$

holds. In order to describe a weaker condition which ensures that $\mathbf{1}_{\Xi_{\bar{O}}} \in$ $\dot{B} V_{\text {loc }}$, we introduce the Riesz (and logarithmic) capacities on $\mathbb{R}^{d}$. For a 
Borel set $A$ of $\mathbb{R}^{d}$, let $\mathscr{P}(A)$ denote the set of all Borel probability measures on $A$. For $\beta \geq 0$, the $\beta$-capacity $\operatorname{Cap}_{\beta}(A)$ of $A$ is defined as

$$
\operatorname{Cap}_{\beta}(A)=\left(\inf _{\lambda \in \mathscr{P}(A)} \iint_{A \times A} g_{\beta}\left(|x-y|_{\mathbb{R}^{d}}\right) \lambda(d x) \lambda(d y)\right)^{-1},
$$

where $g_{\beta}(t)=t^{-\beta}$ for $\beta>0$ and $g_{0}(t)=\log \left(t^{-1} \vee e\right)$. For $\beta<0$, we define $\operatorname{Cap}_{\beta}(A)=1$ for $A \neq \emptyset$ and $\operatorname{Cap}_{\beta}(\emptyset)=0$. These are Choquet capacities.

For $\beta \geq 0$, let $\mathscr{H}^{\beta}(A)$ denote the $\beta$-dimensional Hausdorff measure of $A \subset \mathbb{R}^{d}$. It is known that, if a Borel set $A$ of $\mathbb{R}^{d}$ satisfies $\mathscr{H}^{\beta}(A)<\infty$, then $\operatorname{Cap}_{\beta}(A)=0$ (see, e.g., [3, $\S 1$, Theorem 1]).

For $r>0$, a closed subset $\Sigma_{r}$ of $\mathbb{R}^{d}$ is defined as

$$
\Sigma_{r}=\overline{\{y \in \partial O \mid \delta(y)<r\}} .
$$

Denote $\bigcap_{r>0} \Sigma_{r}$ by $\Sigma$. The following is the main theorem of this section.

Theorem 11. Suppose that

$$
\operatorname{Cap}_{d-4}(\Sigma)=0
$$

Then, $\mathbf{1}_{\Xi_{\bar{O}}} \in \dot{B} V_{\text {loc }} \cap Q R$. In particular, the conclusion of Theorem 10 holds with $\rho=\mathbf{1}_{\Xi_{\bar{O}}}$. Moreover, $\operatorname{Cap}^{\mathbf{1}_{\Xi} \Xi_{\bar{O}}}\left(\partial \Xi_{\bar{O}} \backslash \partial^{\prime} \Xi_{\bar{O}}\right)=0$.

\section{Remark 12.}

(i) The set $\Xi_{\bar{O}}$ coincides with the closure of $\Xi_{O}$ in $E$.

(ii) If $d \geq 4$ and there exists a sequence of Borel subsets $\left\{B_{n}\right\}_{n=1}^{\infty}$ of $\mathbb{R}^{d}$ such that $\bigcup_{n=1}^{\infty} B_{n}=\mathbb{R}^{d}$ and $\mathscr{H}^{d-4}\left(\Sigma \cap B_{n}\right)<\infty$ for every $n \in \mathbb{N}$, then (10) holds.

(iii) Since $\inf _{z \in \partial O} \delta(z)>0$ implies $\Sigma=\emptyset$, the uniform exterior condition (8) implies condition (10).

(iv) The diffusion $\left\{X_{t}\right\}$ associated with $\left(\mathcal{E}^{\rho}, \mathcal{F}^{\rho}\right)$ for $\rho=\mathbf{1}_{\Xi_{\bar{O}}}$ is regarded as the modified reflecting Ornstein-Uhlenbeck process on $\Xi_{\bar{O}}$; the term "modified" is added since the domain $\mathcal{F}^{\rho}$ is defined as the closure of smooth functions and it is not clear whether $\mathcal{F}^{\rho}$ is the maximal domain.

(v) The fact that $\operatorname{Cap}^{\mathbf{1} \Xi_{\bar{O}}}\left(\partial \Xi_{\bar{O}} \backslash \partial^{\prime} \Xi_{\bar{O}}\right)=0$ implies that the measure $\left\|D \mathbf{1}_{\Xi_{\bar{O}}}\right\|$ concentrates on $\partial^{\prime} \Xi_{\bar{O}}$, which means that the process $\left\{X_{t}\right\}$ reflects only at $\partial^{\prime} \Xi_{\bar{O}}$ in view of (7).

Example 13. Let $\varphi:[1,2) \rightarrow \mathbb{R}_{+}$be a convex function such that $\varphi(1)=0$ and $\varphi(t)>0$ for $t \in(1,2)$. Suppose $d \geq 4$ and consider an open subset $O$ of $\mathbb{R}^{d}$ that is defined by

$O=B(0,2) \backslash\left\{\left(x_{1}, \ldots, x_{d}\right) \in \mathbb{R}^{d} \mid x_{d} \in[1,2)\right.$ and $\left.\varphi\left(x_{d}\right)^{2} \geq x_{1}^{2}+\cdots+x_{d-1}^{2}\right\}$. 
Then $\Sigma=\{(0, \ldots, 0,1)\}$ and $\mathscr{H}^{d-4}(\Sigma)<\infty$. Therefore, $\mathbf{1}_{\Xi_{\bar{\sigma}}} \in \dot{B V_{\text {loc }}}$ from Theorem 11 and Remark 12 (ii). It is likely that $\mathbf{1}_{\Xi_{\bar{O}}} \notin B V$ in general, but I have no proof to offer at the moment.

The capacity $\mathrm{Cap}_{d-4}$ is involved in Theorem 11 for the following reason. From Theorem 1.1 of [10] and the arguments in Section 7 in that paper, for $a \in(0, T]$ and $M>0$, there exists a constant $C>0$ depending only on $a$ and $M$ such that, for any closed set $A$ in $\bar{B}(0, M) \subset \mathbb{R}^{d}$,

$$
\begin{aligned}
C^{-1} \operatorname{Cap}_{d-4}(A) & \leq \mathbf{P}\left(\left\{Z_{s}(t) \in A \text { for some }(s, t) \in[a, T] \times[a, T]\right\}\right) \\
& \leq C \operatorname{Cap}_{d-4}(A),
\end{aligned}
$$

where $\left(\left\{Z_{s}\right\}_{s \geq 0}, \mathbf{P}\right)$ is the Ornstein-Uhlenbeck process on $E$ with initial distribution $\mu$. This implies the following property.

If a closed subset $A$ of $\mathbb{R}^{d} \backslash\{0\}$ satisfies $\operatorname{Cap}_{d-4}(A)=0$, then

$$
\operatorname{Cap}\left(\Theta_{A}\right)=0 \text {. }
$$

In particular, since $0 \notin \Sigma$, condition (10) implies

$$
\operatorname{Cap}\left(\Theta_{\Sigma}\right)=0 .
$$

For the proof of Theorem 11, we provide some quantitative estimates as in $[13 ; 9]$. We define a Lipschitz continuous function $q$ on $\mathbb{R}^{d}$ by

$$
q(x)=\inf _{y \in O^{c}}|x-y|_{\mathbb{R}^{d}}-\inf _{y \in O}|x-y|_{\mathbb{R}^{d}}, \quad x \in \mathbb{R}^{d} .
$$

For $r \geq 0$, set $O_{r}=\left\{x \in \mathbb{R}^{d} \mid q(x)>r\right\}$. Note that $O_{0}=O$ and $\{q(x) \geq 0\}=\bar{O}$.

Let $W=C\left([0, \infty) \rightarrow \mathbb{R}^{d}\right)$ and $\mathcal{B}(W)$ be the Borel $\sigma$-field of $W$. Let $\left\{\hat{P}_{x}\right\}_{x \in \mathbb{R}^{d}}$ be the probability measures on $W$ such that the coordinate process $\left\{\omega_{t}\right\}_{t \geq 0}$ is the $d$-dimensional Brownian motion starting at $x$ under $\hat{P}_{x}$ for each $x \in \mathbb{R}^{d}$. For $t \geq 0$, let $\hat{\mathcal{F}}_{t}$ denote the $\sigma$-field generated by $\left\{\omega_{s} \mid s \in[0, t]\right\}$. For an $\left\{\hat{\mathcal{F}}_{t}\right\}$-stopping time $\tau$, define $\hat{\mathcal{F}}_{\tau}$ as $\left\{A \in \mathcal{B}(W) \mid A \cap\{\tau \leq t\} \in \hat{\mathcal{F}}_{t}\right.$ for all $\left.t \geq 0\right\}$. We denote the integral with respect to $\hat{P}_{x}$ by $\hat{E}_{x}$. For $s>0$, the shift operator $\theta_{s}: W \rightarrow W$ is defined by $\left(\theta_{s} \omega\right)_{t}=\omega_{s+t}, t \geq 0$.

The following claims (Lemma 14-Proposition 17) are slight modifications of those in [9].

Lemma 14. Let $x \in \bar{O}$ and choose $y \in \partial O$ such that $q(x)=|x-y|_{\mathbb{R}^{d}}$. Suppose that $\delta(y)>0$. Let $\delta \in(0, \delta(y))$ and take $z \in O^{c}$ such that $B(z, \delta) \cap$ 
$\bar{O}=\{y\}$. Let $C_{\delta}=(d-1) /(2 \delta)$ and $R_{t}=\left|\omega_{t}-z\right|_{\mathbb{R}^{d}}$ for $\omega=\left\{\omega_{t}\right\} \in W$. Then, for each $u>0$,

$\left\{R_{t} \geq \delta\right.$ for all $\left.t \in[0, u]\right\} \subset\left\{R_{t} \leq q(x)+\delta+C_{\delta} t+S_{t}\right.$ for all $\left.t \in[0, u]\right\}$

up to a $\hat{P}_{x}$-null set. Here, $S_{t}$ is the 1-dimensional Brownian motion under $\hat{P}_{x}$ starting at 0 that is defined by

$S_{t}(\omega)=\sum_{i=1}^{d} \int_{0}^{t} \frac{\omega_{s}^{(i)}-z^{(i)}}{R_{s}} d \omega_{s}^{(i)}, \quad \omega_{s}=\left(\omega_{s}^{(1)}, \ldots, \omega_{s}^{(d)}\right), z=\left(z^{(1)}, \ldots, z^{(d)}\right)$,

up to the $\left\{\hat{\mathcal{F}}_{t}\right\}$-stopping time $\inf \left\{t \geq 0 \mid R_{t}=0\right\}$.

Proof. Define $\sigma=\inf \left\{t \geq 0 \mid R_{t}=0\right\}$. Note that $R_{0}=|x-z|_{\mathbb{R}^{d}}=q(x)+\delta$ $\hat{P}_{x}$-a.e. By virtue of Itô's formula,

$$
R_{t}=q(x)+\delta+\int_{0}^{t} \frac{d-1}{2 R_{s}} d s+S_{t} \quad \text { on }\{t<\sigma\} \quad \hat{P}_{x^{-a} \text {.e. }}
$$

Therefore, the assertion holds.

Proposition 15. In the same situation as in Lemma 14, for every $u>0$,

$$
\hat{P}_{x}\left[\inf _{t \in[0, u]} q\left(\omega_{t}\right) \geq 0\right] \leq\left(\frac{d-1}{\delta(y)}+u^{-1 / 2}\right) q(x) .
$$

Proof. Take $\delta \in(0, \delta(y))$. From Lemma 14,

$\hat{P}_{x}\left[R_{t} \geq \delta\right.$ for all $\left.t \in[0, u]\right] \leq \hat{P}_{x}\left[q(x)+\delta+C_{\delta} t+S_{t} \geq \delta\right.$ for all $\left.t \in[0, u]\right]$.

Let $r>q(x)$ and define $\eta=\inf \left\{t \geq 0 \mid C_{\delta} t+S_{t} \leq-r\right\}$. The law of $\eta$ under $\hat{P}_{x}$ is given by

$$
\hat{P}_{x}[\eta \in d t]=\mathbf{1}_{(0, \infty)}(t) \frac{r}{\sqrt{2 \pi t^{3}}} \exp \left(-\frac{\left(r+C_{\delta} t\right)^{2}}{2 t}\right) d t+\left(1-e^{-2 C_{\delta} r}\right) \delta_{\infty}(d t),
$$

where $\delta_{\infty}$ is the delta measure at $\infty$ (see, e.g., [1, p. 295]). Then, we have

$$
\begin{aligned}
\hat{P}_{x}\left[\inf _{t \in[0, u]} q\left(\omega_{t}\right) \geq 0\right] & \leq \hat{P}_{x}\left[R_{t} \geq \delta \text { for all } t \in[0, u]\right] \leq \hat{P}_{x}[\eta>u] \\
& =\int_{u}^{\infty} \frac{r}{\sqrt{2 \pi t^{3}}} \exp \left(-\frac{\left(r+C_{\delta} t\right)^{2}}{2 t}\right) d t+1-e^{-2 C_{\delta} r} \\
& \leq \int_{u}^{\infty} \frac{r}{\sqrt{2 \pi t^{3}}} d t+2 C_{\delta} r=\sqrt{\frac{2}{\pi}} \frac{r}{\sqrt{u}}+2 C_{\delta} r .
\end{aligned}
$$

Letting $r \rightarrow q(x)$ and $\delta \rightarrow \delta(y)$, we obtain the claim. 
For $r>0$, define an $\left\{\hat{\mathcal{F}}_{t}\right\}$-stopping time $\tau_{r}$ by $\tau_{r}=\inf \left\{t \geq 0 \mid \omega_{t} \notin O_{r}\right\}$. Let $\hat{P}_{x}^{r}$ be the law of $\tau_{r}$ under $\hat{P}_{x}$. The following lemma is the same as $[9$, Lemma 3.2], so the proof is omitted.

Lemma 16. $\hat{P}_{x}^{r}([0, t])$ is differentiable in $t$ on $(0, \infty)$ and there exists a constant $\hat{C}_{1}>0$ independent of $x, r$ and $t$ such that $\frac{d}{d t} \hat{P}_{x}^{r}([0, t]) \leq \hat{C}_{1} t^{-1}$.

For a closed subset $A$ of $\mathbb{R}^{d}$, a stopping time $\sigma_{A}$ is defined as $\sigma_{A}=\inf \{t \geq$ $\left.0 \mid \omega_{t} \in A\right\}$. For $r>0$, let

$$
A_{r}=\left\{x \in \mathbb{R}^{d} \mid \bar{B}(x, r) \cap \Sigma_{r} \neq \emptyset\right\},
$$

where $\Sigma_{r}$ is defined in (9).

Proposition 17. For $\gamma>0, u>0, r \in(0, \gamma]$, and $x \in \bar{O}$, the inequality

$$
\hat{P}_{x}\left[0 \leq \inf _{t \in[0, u]} q\left(\omega_{t}\right) \leq r, \sigma_{A_{\gamma}}>u\right] \leq\left(\frac{d-1}{\gamma}+\hat{C}_{2} u^{-1 / 2}\right) r
$$

holds with $\hat{C}_{2}=4 \hat{C}_{1}+2$.

Proof. Denote $(d-1) / \gamma$ by $C_{\gamma}^{\prime}$. If $x \in A_{\gamma},(14)$ is trivial since the lefthand side is 0 . Suppose $x \in \bar{O} \backslash\left(O_{r} \cup A_{\gamma}\right)$. Then, $q(x)<r \leq \gamma$. Take $y \in \partial O$ such that $|x-y|_{\mathbb{R}^{d}}=q(x)$. Because $x \notin A_{\gamma}$ and $q(x)<\gamma, y$ does not belong to $\Sigma_{\gamma}$ : that is, $\delta(y) \geq \gamma$. From Proposition 15 ,

$$
\hat{P}_{x}\left[0 \leq \inf _{t \in[0, u]} q\left(\omega_{t}\right) \leq r\right] \leq\left(\frac{d-1}{\delta(y)}+u^{-1 / 2}\right) q(x) \leq\left(C_{\gamma}^{\prime}+u^{-1 / 2}\right) r .
$$

Next, suppose $x \in O_{r} \backslash A_{\gamma}$. Then,

$$
\begin{aligned}
& \left\{0 \leq \inf _{t \in[0, u]} q\left(\omega_{t}\right) \leq r\right\}=\left\{\tau_{r} \leq u, 0 \leq \inf _{t \in\left[0, u-\tau_{r}\right]} q\left(\left(\theta_{\tau_{r}} \omega\right)_{t}\right)\right\} \\
& \subset \bigcup_{k=1}^{\infty}\left\{2^{-k} u<u-\tau_{r} \leq 2^{-k+1} u, 0 \leq \inf _{t \in\left[0,2^{-k} u\right]} q\left(\left(\theta_{\tau_{r}} \omega\right)_{t}\right)\right\} \cup\left\{\tau_{r}=u\right\} .
\end{aligned}
$$

From Lemma $16, \hat{P}_{x}\left[\tau_{r}=u\right]=0$. From the strong Markov property and Proposition 15,

$$
\begin{aligned}
& \hat{P}_{x}\left[2^{-k} u<u-\tau_{r} \leq 2^{-k+1} u, 0 \leq \inf _{t \in\left[0,2^{-k} u\right]} q\left(\left(\theta_{\tau_{r}} \omega\right)_{t}\right), \sigma_{A_{\gamma}}>u \mid \hat{\mathcal{F}}_{\tau_{r}}\right] \\
& \leq \mathbf{1}_{\left\{2^{-k} u<u-\tau_{r} \leq 2^{-k+1} u\right\}} \cdot \hat{E}_{x}\left[\hat{P}_{\omega_{\tau_{r}}}\left[0 \leq \inf _{t \in\left[0,2^{-k} u\right]} q\left(\omega_{t}\right)\right] ; \omega_{\tau_{r}} \in \partial O_{r} \backslash A_{\gamma}\right] \\
& \leq \mathbf{1}_{\left\{2^{-k} u<u-\tau_{r} \leq 2^{-k+1} u\right\}} \cdot\left(C_{\gamma}^{\prime}+\left(2^{-k} u\right)^{-1 / 2}\right) r \\
& \leq \mathbf{1}_{\left\{2^{-k} u<u-\tau_{r} \leq 2^{-k+1} u\right\}} \cdot\left(C_{\gamma}^{\prime}+\left(\left(u-\tau_{r}\right) / 2\right)^{-1 / 2}\right) r .
\end{aligned}
$$


Therefore,

$$
\begin{aligned}
& \hat{P}_{x}\left[0 \leq \inf _{t \in[0, u]} q\left(\omega_{t}\right) \leq r, \sigma_{A_{\gamma}}>u\right] \\
& \leq r \hat{E}_{x}\left[C_{\gamma}^{\prime}+\left(\left(u-\tau_{r}\right) / 2\right)^{-1 / 2} ; \tau_{r} \leq u\right] \\
& \leq r C_{\gamma}^{\prime}+r\left(\frac{u}{4}\right)^{-1 / 2} \hat{P}_{x}\left[\tau_{r} \leq u / 2\right]+r \int_{u / 2}^{u}\left(\frac{u-s}{2}\right)^{-1 / 2} \hat{C}_{1} s^{-1} d s
\end{aligned}
$$

(from Lemma 16)

$$
\begin{aligned}
& \leq r C_{\gamma}^{\prime}+2 r u^{-1 / 2}+\frac{2 \hat{C}_{1} r}{u} \int_{u / 2}^{u}\left(\frac{u-s}{2}\right)^{-1 / 2} d s \\
& =r C_{\gamma}^{\prime}+2 r u^{-1 / 2}+4 \hat{C}_{1} r u^{-1 / 2} .
\end{aligned}
$$

This completes the proof.

Lemma 18. Let $\left\{B_{n}\right\}_{n=1}^{\infty}$ be a decreasing sequence of closed subsets of $\mathbb{R}^{d}$. If $\operatorname{Cap}_{d-4}\left(\bigcap_{n=1}^{\infty} B_{n}\right)=0$, then $\operatorname{Cap}\left(\Theta_{B_{n}}\right)$ converges to 0 as $n \rightarrow \infty$.

Proof. From (11), $\operatorname{Cap}\left(\Theta_{\bigcap_{n=1}^{\infty} B_{n}}\right)=0$. For $\varepsilon>0$, take a compact subset $F$ of $E$ such that $\operatorname{Cap}(E \backslash F)<\varepsilon$. Since $\left\{\Theta_{B_{n}} \cap F\right\}_{n=1}^{\infty}$ is a decreasing sequence of compact sets,

$$
\lim _{n \rightarrow \infty} \operatorname{Cap}\left(\Theta_{B_{n}} \cap F\right)=\operatorname{Cap}\left(\Theta_{\bigcap_{n=1}^{\infty} B_{n}} \cap F\right)=0 .
$$

Therefore,

$$
\lim _{n \rightarrow \infty} \operatorname{Cap}\left(\Theta_{B_{n}}\right) \leq \lim _{n \rightarrow \infty} \operatorname{Cap}\left(\Theta_{B_{n}} \cap F\right)+\operatorname{Cap}(E \backslash F) \leq \varepsilon .
$$

Since $\varepsilon>0$ is arbitrary, we obtain the claim.

Proof of Theorem 11. Recall the definition of $A_{r}$ in (13). We note that

$$
\bigcap_{k=1}^{\infty} A_{1 / k}=\Sigma .
$$

From Proposition 17 and (12),

$$
\begin{aligned}
\mu\left(\Xi_{\bar{O}} \backslash \Xi_{O}\right) & =\hat{P}_{0}\left[\inf _{t \in[0, T]} q\left(\omega_{t}\right)=0\right] \\
& \leq \lim _{n \rightarrow \infty} \hat{P}_{0}\left[\left\{\inf _{t \in[0, T]} q\left(\omega_{t}\right)=0\right\} \cap\left\{\sigma_{A_{1 / n}}>T\right\}\right]+\mu\left(\Theta_{\Sigma}\right) \\
& =0 .
\end{aligned}
$$


That is, $\mathbf{1}_{\Xi_{\bar{O}}}=\mathbf{1}_{\Xi_{O}} \mu$-a.e. Since $\mathbf{1}_{\Xi_{O}}$ is a lower semicontinuous function, $\mathbf{1}_{\Xi_{\bar{O}}} \in Q R$ (cf. [5, p. 230]).

For $k \in \mathbb{N}$, let $B_{k}$ denote the closure of $A_{1 / k}^{c}$, and let $F_{k}=\Xi_{B_{k}}$. Then, $\left\{F_{k}\right\}_{k=1}^{\infty}$ is an $\mathcal{E}$-nest from (12), (15), and Lemma 18. Since $A_{1 /(k+1)} \subset B_{k}^{c}$, $\Theta_{A_{1 /(k+1)}} \subset E \backslash F_{k}$ for each $k$. Let $h(w)=\inf _{t \in[0, T]} q(w(t))$ for $w \in E$ and $\chi(t)=(0 \vee t) \wedge 1$ for $t \in \mathbb{R}$. For $m \in \mathbb{N}$, let $f_{m}(w)=\chi(m h(w))$ for $w \in E$. Then, $f_{m} \in \mathbb{D}^{1,1}$ and $f_{m}$ converges to $\mathbf{1}_{\Xi_{O}}$ in $L^{1}$ as $m \rightarrow \infty$. Moreover, for $k \in \mathbb{N}$ and $m \geq k+1$,

$$
\begin{aligned}
\left\|\left(\nabla f_{m}\right) \cdot \mathbf{1}_{F_{k}}\right\|_{1} & \leq \int_{F_{k}} m \cdot \mathbf{1}_{\{0 \leq h \leq 1 / m\}} d \mu \\
& \leq m \mu\left(\{0 \leq h \leq 1 / m\} \backslash \Theta_{A_{1 /(k+1)}}\right) \\
& \leq(k+1)(d-1)+\hat{C}_{2} T^{-1 / 2} \quad \text { (from Proposition 17). }
\end{aligned}
$$

Therefore, $\sup _{m \in \mathbb{N}}\left\|\left(\nabla f_{m}\right) \cdot \mathbf{1}_{F_{k}}\right\|_{1}<\infty$ for each $k \in \mathbb{N}$. From Theorem 3, we conclude that $\mathbf{1}_{\Xi_{\bar{\delta}}} \in B V_{\text {loc }}$.

The identity $\operatorname{Cap}^{1} \Xi_{\bar{O}}\left(\partial \Xi_{\bar{O}} \backslash \partial^{\prime} \Xi_{\bar{O}}\right)=0$ is proved in a similar way to the proof of [9, Theorem 2.4]. We provide details for the readers' convenience. Take an $\mathcal{E}$-nest $\left\{F_{k}^{\prime}\right\}_{k=1}^{\infty}$ and functions $\left\{\varphi_{k}\right\}_{k=1}^{\infty}$ obtained by applying Lemma 4 to the $\mathcal{E}$-nest $\left\{F_{k}\right\}_{k=1}^{\infty}$ defined above. For $s \in(0, T)$, we define a closed set $V_{s}$ by

$$
V_{s}=\left\{w \in E \mid \inf _{t \in[0, s]} q(w(t))=0 \text { and } \inf _{t \in[s, T]} q(w(t))=0\right\} .
$$

Then, $\partial \Xi_{\bar{O}} \backslash \partial^{\prime} \Xi_{\bar{O}} \subset \bigcup_{s \in(0, T) \cap \mathbb{Q}} V_{s}$. Fix $s \in(0, T)$ and define a map $f: E \rightarrow \mathbb{R}^{2}$ by

$$
f(w)=\left(\inf _{t \in[0, s]} q(w(t)), \inf _{t \in[s, T]} q(w(t))\right) .
$$

Fix $k \in \mathbb{N}$ and take $l \in \mathbb{N}$ such that $\varphi_{k} \in \mathbb{D}_{F_{l}}^{1,2}$. Let $\varepsilon>0$. Henceforth, $C$ denotes an unimportant positive constant independent of $\varepsilon$ that may vary line by line. Take a smooth function $g$ on $[0, \infty)$ such that

$$
g(t)= \begin{cases}1 & t \in\left[0, e^{-2 / \varepsilon}\right], \\ -3 \varepsilon \log t-4 & t \in\left[e^{-14 /(9 \varepsilon)}, e^{-13 /(9 \varepsilon)}\right], \\ 0 & t \in\left[e^{-1 / \varepsilon}, \infty\right)\end{cases}
$$

and $-3 \varepsilon / t \leq g^{\prime}(t) \leq 0$ for all $t>0$. We define a function $\zeta: \mathbb{R}^{2} \rightarrow \mathbb{R}$ by $\zeta(x, y)=\sqrt{x^{2}+y^{2}}$ and set $\iota=g \circ \zeta$. Since $\iota \circ f$ is a bounded $H$-Lipschitz 
continuous function, it belongs to $\mathbb{D}_{b}^{1,2}$. Let $\eta=(\iota \circ f) \varphi_{k}+e_{E \backslash F_{k}^{\prime}} \in \mathbb{D}^{1,2}$. Then, $\eta \geq 1 \mu$-a.e. on some open set including $V_{s}$. Since $(\iota \circ f) \varphi_{k} \in \mathbb{D}_{F_{l}}^{1,2}$,

$$
\begin{aligned}
& \mathcal{E}^{\mathbf{1}_{\Xi_{\bar{O}}}}\left(\left.\eta\right|_{\Xi_{\bar{O}}},\left.\eta\right|_{\Xi_{\bar{O}}}\right) \\
& \leq 2 \mathcal{E}^{\boldsymbol{1}_{\bar{O}}}\left(\left.\left((\iota \circ f) \varphi_{k}\right)\right|_{\Xi_{\bar{O}}},\left.\left((\iota \circ f) \varphi_{k}\right)\right|_{\Xi_{\bar{O}}}\right)+2 \mathcal{E}^{\mathbf{1}_{\bar{O}}}\left(\left.e_{E \backslash F_{k}^{\prime}}\right|_{\Xi_{\bar{O}}},\left.e_{E \backslash F_{k}^{\prime}}\right|_{\Xi_{\bar{O}}}\right) \\
& \leq 2 \int_{\Xi_{\bar{O} \cap F_{l}}}|\nabla(\iota \circ f)|_{H}^{2} d \mu+4 \mathcal{E}\left(\varphi_{k}, \varphi_{k}\right)+2 \mathcal{E}\left(e_{E \backslash F_{k}^{\prime}}, e_{E \backslash F_{k}^{\prime}}\right) .
\end{aligned}
$$

Denoting the gradient operator on $\mathbb{R}^{2}$ by $\nabla_{\mathbb{R}^{2}}$, we have

$$
\begin{aligned}
& \int_{\Xi_{\bar{O}} \cap F_{l}}|\nabla(\iota \circ f)|_{H}^{2} d \mu=\int_{\Xi_{\bar{O} \cap B_{l}}}\left|\left\langle(\nabla f)(w),\left(\nabla_{\mathbb{R}^{2}} \iota\right)(f(w))\right\rangle_{\mathbb{R}^{2}}\right|_{H}^{2} \mu(d w) \\
& \leq C \int_{\Xi_{\bar{O} \cap B_{l}}}\left|\left(\nabla_{\mathbb{R}^{2}} \iota\right)(f(w))\right|_{\mathbb{R}^{2}}^{2} \mu(d w) \\
& =C \int_{\left\{(x, y) \in \mathbb{R}^{2} \mid x \geq 0, y \geq 0\right\}}\left|\nabla_{\mathbb{R}^{2}} \iota\right|_{\mathbb{R}^{2}}^{2} d\left(f_{*}\left(\left.\mu\right|_{\Xi_{\bar{O} \cap B_{l}}}\right)\right) \\
& =: I_{1} \text {. }
\end{aligned}
$$

In the first line, $\langle\cdot, \cdot\rangle_{\mathbb{R}^{2}}$ denotes a pairing between elements in $H \otimes \mathbb{R}^{2}$ and in $\mathbb{R}^{2}$ that takes values in $H$. We note that

$$
\begin{aligned}
\left|\nabla_{\mathbb{R}^{2}} \iota\right|_{\mathbb{R}^{2}}^{2} & =(\partial \iota / \partial x)^{2}+(\partial \iota / \partial y)^{2} \\
& =\left(g^{\prime} \circ \zeta(x, y)\right)^{2} \frac{x^{2}}{x^{2}+y^{2}}+\left(g^{\prime} \circ \zeta(x, y)\right)^{2} \frac{y^{2}}{x^{2}+y^{2}} \\
& =\left(g^{\prime} \circ \zeta(x, y)\right)^{2} .
\end{aligned}
$$

By letting $\psi=(\zeta \circ f)_{*}\left(\left.\mu\right|_{\Xi_{\bar{O} \cap B_{l}}}\right)$, we obtain

$$
I_{1}=C \int_{0}^{\infty} g^{\prime}(r)^{2} \psi(d r) \leq 9 C \varepsilon^{2} \int_{e^{-2 / \varepsilon}}^{e^{-1 / \varepsilon}} r^{-2} \psi(d r)=: I_{2} .
$$

We now have

$$
\begin{aligned}
\Psi(r) & :=\psi([0, r])=\left(f_{*}\left(\left.\mu\right|_{\Xi_{\bar{O} \cap B_{l}}}\right)\right)\left(\zeta^{-1}([0, r])\right) \\
& =\mu\left[\left\{\begin{array}{l|l}
w \in \Xi_{\bar{O} \cap B_{l}} \mid \inf _{t \in[0, s]} q(w(t))^{2}+\inf _{t \in[s, T]} q(w(t))^{2} \leq r^{2}
\end{array}\right\}\right] \\
& \leq \mu\left[\left\{\begin{array}{l|l}
w \in \Xi_{\bar{O} \backslash A_{1 /(l+1)}} & \begin{array}{l}
0 \leq \inf _{t \in[0, s]} q(w(t)) \leq r, \\
0 \leq \inf _{t \in[s, T]} q(w(t)) \leq r
\end{array}
\end{array}\right]\right.
\end{aligned}
$$




$$
\begin{gathered}
\leq \hat{E}_{0}\left[\hat{P}_{\omega_{s}}\left[0 \leq \inf _{t \in[0, T-s]} q\left(\omega_{t}\right) \leq r, \sigma_{A_{1 /(l+1)}}>T-s\right] ;\right. \\
\left.0 \leq \inf _{t \in[0, s]} q\left(\omega_{t}\right) \leq r, \sigma_{A_{1 /(l+1)}}>s\right] .
\end{gathered}
$$

From Proposition 17, if $r \leq 1 /(l+1)$, this expectation is dominated by

$$
\begin{aligned}
& \left((d-1)(l+1)+C(T-s)^{-1 / 2}\right) r \hat{P}_{0}\left[0 \leq \inf _{t \in[0, s]} q\left(\omega_{t}\right) \leq r, \sigma_{A_{1 /(l+1)}}>s\right] \\
& \leq\left((d-1)(l+1)+C(T-s)^{-1 / 2}\right)\left((d-1)(l+1)+C s^{-1 / 2}\right) r^{2} \\
& =C r^{2} .
\end{aligned}
$$

Thus, for $\varepsilon \leq 1 / \log (l+1)$,

$$
\begin{aligned}
I_{2} & =9 C \varepsilon^{2} \int_{e^{-2 / \varepsilon}}^{e^{-1 / \varepsilon}} r^{-2} d \Psi(r) \\
& =C \varepsilon^{2}\left\{\left[\frac{\Psi(r)}{r^{2}}\right]_{e^{-2 / \varepsilon}}^{e^{-1 / \varepsilon}}+\int_{e^{-2 / \varepsilon}}^{e^{-1 / \varepsilon}} \frac{2 \Psi(r)}{r^{3}} d r\right\} \\
& \leq C \varepsilon^{2}\left(C+\int_{e^{-2 / \varepsilon}}^{e^{-1 / \varepsilon}} \frac{C}{r} d r\right)=C \varepsilon^{2}(1+1 / \varepsilon) .
\end{aligned}
$$

Therefore,

$$
\begin{aligned}
\operatorname{Cap}^{1 \Xi_{\bar{O}}}\left(V_{s}\right) \leq & \mathcal{E}^{1 \Xi_{\bar{O}}}\left(\left.\eta\right|_{\Xi_{\bar{O}}},\left.\eta\right|_{\Xi_{\bar{O}}}\right)+\left\|\left.\eta\right|_{\Xi_{\bar{O}}}\right\|_{L^{2}\left(\left.\mu\right|_{\Xi_{\bar{O}}}\right)}^{2} \\
\leq & 2 \int_{\Xi_{\bar{O}} \cap F_{l}}|\nabla(\iota \circ f)|_{H}^{2} d \mu+4 \mathcal{E}\left(\varphi_{k}, \varphi_{k}\right)+2 \mathcal{E}\left(e_{E \backslash F_{k}^{\prime}}, e_{E \backslash F_{k}^{\prime}}\right) \\
& +2 \Psi\left(e^{-1 / \varepsilon}\right)+2\left\|e_{E \backslash F_{k}^{\prime}}\right\|_{2}^{2} \\
\leq & C \varepsilon^{2}(1+1 / \varepsilon)+4 \mathcal{E}\left(\varphi_{k}, \varphi_{k}\right)+2 \operatorname{Cap}\left(E \backslash F_{k}^{\prime}\right)+C e^{-2 / \varepsilon} .
\end{aligned}
$$

By letting $\varepsilon \rightarrow 0$ and then $k \rightarrow \infty$, we obtain $\operatorname{Cap}^{1} \bar{\sigma}_{\bar{\sigma}}\left(V_{s}\right)=0$. Therefore,

$$
\operatorname{Cap}^{{ }^{1} \Xi_{\bar{O}}}\left(\partial \Xi_{\bar{O}} \backslash \partial^{\prime} \Xi_{\bar{O}}\right) \leq \sum_{s \in(0, T) \cap \mathbb{Q}} \operatorname{Cap}^{{ }^{1} \Xi_{\bar{O}}}\left(V_{s}\right)=0 .
$$

Remark 19. In this section, we considered only one-sided pinned path spaces as the underlying space for simplicity. However, the general idea of the argument is also valid for pinned path spaces, as discussed in [9].

\section{Acknowledgements}

This research was partially supported by JSPS KAKENHI Grant Number 24540170 . 


\section{References}

1. Borodin, A. N. and Salminen, P., Handbook of Brownian motion-facts and formulae, 2nd ed., Probability and its Applications, Birkhäuser Verlag, Basel, 2002.

2. Bouleau, N. and Hirsch, F., Dirichlet forms and analysis on Wiener space, de Gruyter Studies in Mathematics 14, Walter de Gruyter, Berlin, 1991.

3. Carleson, L., Selected problems on exceptional sets, Van Nostrand Mathematical Studies 13, D. Van Nostrand Co., Inc., Princeton, N.J.-Toronto, Ont.-London, 1967.

4. Fukushima, M., On semimartingale characterizations of functionals of symmetric Markov processes, Electron. J. Probab. 4 (1999), no. 18, pp. 1-32.

5. Fukushima, M., $B V$ functions and distorted Ornstein Uhlenbeck processes over the abstract Wiener space, J. Funct. Anal. 174 (2000), 227-249.

6. Fukushima, M. and Hino, M., On the space of BV functions and a related stochastic calculus in infinite dimensions, J. Funct. Anal. 183 (2001), 245268.

7. Fukushima, M., Oshima, Y., and Takeda, M., Dirichlet forms and symmetric Markov processes, 2nd ed., de Gruyter Studies in Mathematics 19, Walter de Gruyter, Berlin, 2011.

8. Hino, M., Integral representation of linear functionals on vector lattices and its application to BV functions on Wiener space, in: Stochastic Analysis and Related Topics in Kyoto in honour of Kiyosi Itô, 121-140, Advanced Studies in Pure Mathematics 41, 2004.

9. Hino, M. and Uchida, H., Reflecting Ornstein-Uhlenbeck processes on pinned path spaces, in: Proceedings of RIMS Workshop on Stochastic Analysis and Applications, 111-128, RIMS Kôkyûroku Bessatsu B6, Res. Inst. Math. Sci. (RIMS), Kyoto, 2008.

10. Khoshnevisan, D. and Shi, Z., Brownian sheet and capacity, Ann. Probab. 27 (1999), 1135-1159.

11. Nualart, D., The Malliavin calculus and related topics, 2nd ed., Probability and its Applications, Springer-Verlag, Berlin, 2006.

12. Röckner, M. and Schmuland, B., Tightness of general $C_{1, p}$ capacities on Banach space, J. Funct. Anal. 108 (1992), 1-12.

13. Uchida, H., Reflecting Ornstein-Uhlenbeck processes on path spaces (in Japanese), Master thesis in Kyoto University, 2006. 